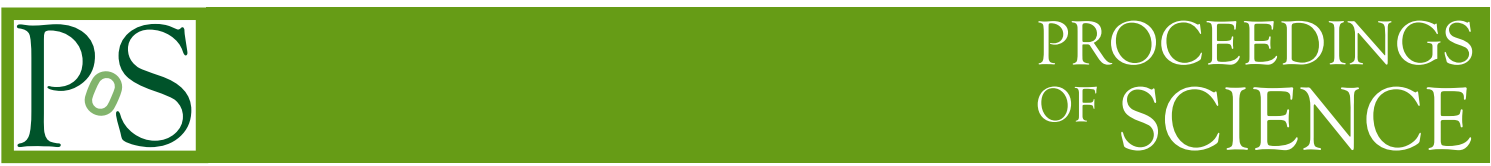

\title{
QED Corrections to Hadronic Observables
}

\section{Agostino Patella*}

Theoretical Physics Department, CERN, Geneva, Switzerland

Centre for Mathematical Sciences, Plymouth University, Plymouth, PLA 8AA, UK

E-mail: agostino.patella@cern.ch

\begin{abstract}
When aiming at the percent precision in hadronic quantities calculated by means of lattice simulations, isospin breaking effects become relevant. These are of two kinds: up/down mass splitting and electromagnetic corrections. In order to account properly for the latter, a consistent formulation of electrically-charged states in finite volume is needed. In fact on a periodic torus Gauss law and large gauge transformations forbid the propagation of electrically-charged states. In this talk I will review methods that have been used or proposed so far in order to circumvent this problem, while highlighting practical as well as conceptual pros and cons. I will also review and discuss various methods to calculate electromagnetic corrections to hadron masses and decay rates in numerical simulations.
\end{abstract}

This is a reduced version of the contribution, edited in order to comply with the PoS page limit. Discussions have been shortened, conclusions and appendices omitted. Please refer to the arXiv for the full version.

CERN-TH-2017-019

34th annual International Symposium on Lattice Field Theory

24-30 July 2016

University of Southampton, UK

${ }^{*}$ Speaker. 


\section{Introduction}

Isospin is an approximate symmetry violated at the percent level by two effects: up and down-quark mass difference and electromagnetic interactions. There are three main reasons to include isospinbreaking effects in lattice simulations:

- Calculate pure isospin-breaking effect, like hadron-mass splitting or up and down-quark renormalized mass difference.

The lattice community has been active on the front of hadron-mass splitting since the exploratory studies in the electroquenched setup in [1, 2, 3, 4]. The 2015 BMW work [5] on baryon-mass splitting remains a landmark for the complexity of the simulations and for the rigorous treatment of finite-volume effects in presence of dynamical photons. The QCDSFCSSM-RIKEN-Kobe (in the following, QCDSF for short) collaboration has reported in this conference on their hadron-mass splitting calculation [6, 7, 8]. The BMW and QCDSF work has been reviewed Liu's plenary talk in this conference and related proceedings [9].

The BMW collaboration has also reported in this conference on their calculation of the up and down-quark mass difference, in the electroquenched setup [10,11]. The $m_{u}=0$ solution to the strong CP problem is excluded by their calculation, assuming that the estimate for the systematic error due to electroquenching be reliable.

All calculations mentioned so far of the BMW and QCDSF collaborations rely on non-local prescriptions to describe charged particles. I will come back to this point later on.

- Improve on the determination of observable, which have reached the precision of order $1 \%$. This is the case for decay rates of light mesons.

In fact the FLAG working group reports the following world averages for pion and kaon decay constants at the isosymmetric point [12]

$$
F_{\pi}=130.2(1.4) \mathrm{MeV}, \quad F_{K}=155.6(0.4) \mathrm{MeV},
$$

with a relative error of $1 \%$ and $0.3 \%$ respectively. On the other hand radiative corrections to leptonic decay rates of pion and kaon are estimated to be about $1.8 \%$ and $1.1 \%$ respectively on the basis of $\chi \mathrm{PT}[13,14]$, and already dominate the error budget. In order to improve on the state-of-the-art lattice determinations of such decay rates, isospin breaking corrections must be included.

The RM-SOTON collaboration has presented in this conference a strategy to calculate leptonic decay rates, and some preliminary results for light mesons in the electroquenched setup [15, 16, 17, 18]. Calculations of radiative correction to decay rates present extra complications with respect e.g. to mass-splitting calculations due to the existence of IR divergences in QED. Even though IR divergences have been studied since the early days of QED and are very well understood, they do not belong to the standard toolbox of the lattice community. For this reason I have chosen to devote section 3 to reviewing general aspects of decay rates and IR divergences, in relation to the RM-SOTON calculation, but also with a (superficial) eye to heavy-meson decay rates.

- Improve on theoretical estimates of possibly large radiative corrections, like to decay rates of heavy mesons, or to the HVP contribution to $g_{\mu}-2$. 
The RBC-UKQCD collaboration has presented in this conference some preliminary studies on the isospin-breaking correction to the HVP contribution to $g_{\mu}-2$ [19].

On the other hand no activity has been reported on equally-interesting radiative corrections to decay rates of heavy mesons. For instance radiative corrections to $B \rightarrow D \ell v$ are relevant for a precise determination of $\left|V_{c b}\right|$ at the upcoming Belle II experiment (see for instance BELLE2-NOTE-PH-2015-002), and are expected to be of order 3\% as reported in the 2016 PDG review [20]. A search in the references therein (e.g. [21, 22, 23, 24, 25]) reveals that radiative corrections are estimated very crudely by separating three contributions:

1. Short-distance contributions, due to photons coupling to the $W$. These contributions can be systematically accounted for by means of the OPE.

2. Long-distance soft-photon contributions (inner-bremsstrahlung) in loops and finale-state radiation. These are analytically calculable.

3. Long-distance hard-photon contributions (structure-dependent contributions). These are fully non-perturbative, and they are either neglected or estimated by saturating relevant matrix elements with a few resonances (see also [26]).

Structure-dependent contributions may be enhanced by quasi-on-shell resonances and collinear quasi-divergences which arise when charged leptons are produced with large energy, and neglecting them may be not fully justified at the required level of precision (some of these issues will be highlighted in section 3). It is important to understand if a robust strategy can be designed to estimate these contributions from lattice simulations.

Moving to more numerical issues, two very general strategies have been proposed in order to calculate isospin-breaking effects in lattice simulations, which have been already reviewed in detail in plenary talks $[27,28]$ of previous editions of the Lattice conference:

- Generate configurations in the isosymmetric limit and reweight them. The reweighting factor can be included in a standard fashion as a ratios of fermionic determinants averaged over the photon field as originally proposed in [29,30]. Alternatively the reweighting factor (along with the observables) can be expanded at first order in $m_{d}-m_{u}$ and $\alpha_{\mathrm{em}}$ (RM123 method [31, 32]). In practice one needs to insert $\bar{\psi} \psi$ and $\bar{\psi} \gamma_{\mu} \psi$ operators into the original observables, multiply times the photon propagator and sum over the photon momenta. The convolution with the photon propagator can be stochastically estimated. The clear advantage of this particular incarnation of reweighting is that one has direct access to the leading isospin-breaking corrections as $O(1)$ observables, rather than $O\left(\alpha_{\mathrm{em}}\right) \sim O\left(\Delta m_{u d}\right)$ effects on $O(1)$ observables.

- Generate non-isosymmetric configurations at unphysically large values of $m_{d}-m_{u}$ and $\alpha_{e m}$ and extrapolate to the physical point. This strategy has been used by the BWM [5] and the QCDSF collaboration [6] (first explorations in the electroquenched setup can be found in [1]). As observed by the BMW collaboration $O\left(\alpha_{\mathrm{em}}^{2}\right)$ effects seem to be surprisingly small if $\alpha_{\mathrm{em}}$ is the renormalized coupling, and this is essential for the strategy to work. The extrapolation can be performed along different parameter trajectories. For instance the QCDSF collaboration claims [7] that choosing a trajectory that departs symmetrically (in some sense that is specified in their work) from the SU(3) symmetric point is particularly beneficial. 
A detailed comparison between these two methods would be very interesting and is still missing. A first move in this direction has been presented by the RBC-UKQCD collaboration [19] in the context of the electroquenched approximation.

The inclusion of electromagnetic corrections in lattice simulations is especially challenging from the theoretical point of view. Typically lattice simulations assume a finite box with periodic boundary conditions along the spatial directions. However Gauss law forbids states with total electric charge different from zero in a box with periodic boundary conditions. This is an essential obstruction for numerical simulations which aim at calculating properties of charged particles. Several prescriptions have been proposed in order to circumvent this problem. A big fraction of this contribution (section 2) is devoted to a critical review of these prescriptions, what we know about them, together with some new results. In particular I will discuss the following aspects:

- If $\tilde{A}_{\mu}(p)$ is the photon field in Fourier space, the $\mathrm{QED}_{\mathrm{TL}}$ prescription [1] is defined by imposing the constraint $\tilde{A}_{\mu}(0)=0$. As already noticed by BMW [5], $\mathrm{QED}_{\mathrm{TL}}$ does not have a transfer matrix with a regular perturbative expansion. $\mathrm{QED}_{\mathrm{TL}}$ has been used in the $\mathrm{BMW}$ calculation of the up and down-quark mass difference $[10,11])$.

- The $\mathrm{QED}_{\mathrm{SF}}$ prescription [33] is defined by restricting the value of $V^{-1} e \tilde{A}_{\mu}(0)$ in the interval $\left(-\pi L_{\mu}^{-1}, \pi L_{\mu}^{-1}\right)$. Charged-field two-point functions in $\mathrm{QED}_{\mathrm{SF}}$ do not have a local representation in time, therefore the existence of a transfer matrix in the charged sector is not guaranteed (and very unlikely). $\mathrm{QED}_{\mathrm{SF}}$ has been used in all QCDSF calculations.

- The $\mathrm{QED}_{\mathrm{L}}$ prescription [34] is defined by imposing the constraint $\tilde{A}_{\mu}\left(p_{0}, \mathbf{0}\right)=0$ for any $p_{0}$. Renormalization of higher-dimensional operators by local counterterms breaks down in the $\phi^{4}$ scalar theory with a constraint analogous to $\mathrm{QED}_{\mathrm{L}}$. This also implies that the operator product expansion as well as the Symanzik effective-theory description of the lattice theory close to the continuum limit break down because of non-local contributions. $\mathrm{QED}_{\mathrm{L}}$ has been used in the BMW baryon-mass splitting calculation [5] and in the RM123-SOTON decay rate exploratory calculation [17].

- QED with a massive photon $\left(\mathrm{QED}_{\mathrm{m}}\right)$ is a perfectly consistent QFT. In finite volume the theory has two IR regulators, the photon mass $m_{\gamma}$ and the box size $L$ [35]. The $L \rightarrow \infty$ limit has to be carefully taken before the $m_{\gamma} \rightarrow 0$ limit, as the two limits do not commute. For instance, if $m_{\gamma}$ is too small, the spatial-momentum dependence in charged-hadron two-point functions is suppressed and the extraction of states with definite momentum is hindered.

- QED with C-parity boundary conditions $\left(\mathrm{QED}_{\mathrm{C}}\right)$ in the spatial directions is also a perfectly consistent QFT [36, 37]. The boundary conditions partially break flavor conservation in a local fashion. The flavour mixing induced by the boundary conditions does not affect the majority of the stable hadrons, is exponentially suppressed with the volume in two-point functions, and absent in the renormalization of composite operators. QED $_{C}$ also allows a completely gaugeinvariant description of charged interpolating operators.

The prescription that defines $\mathrm{QED}_{\mathrm{L}}$ has been widely believed to be harmless for a few years, on the basis that operators with dimension not larger than 4 renormalize as in infinite volume at one loop. The first sign of sickness was analyzed in [38]: in the non-relativistic limit charged particles and antiparticles do not decouple. The breakdown of the Symanzik expansion casts a shadow on 
the ability to extract a reliable continuum limit, especially when large values of $\alpha_{\mathrm{em}}$ are used in the extrapolation. In favour of $\mathrm{QED}_{\mathrm{L}}$ one may argue that none of the above prescriptions is completely free of problems or potential subtleties. This does not come as a surprise as the constraint that we are trying to circumvent (Gauss law) is not just a technical one, but has a deep physical origin rooted in the very fundamental structure of QED. Reality is that the subtleties arising in local prescriptions as $\mathrm{QED}_{\mathrm{m}}$ and $\mathrm{QED}_{\mathrm{C}}$ can be systematically studied and accounted for with the powerful tools of QFT. On the other hand no general tool exists for non-local prescriptions and every solution to any newly found problem is a patch whose validity may be debatable until such general tools are developed. My personal recommendation is to use and further develop theoretically sound setups, as the only way to eliminate unwanted, uncontrollable and unnecessary systematic errors in calculations which aim the the percent precision.

\section{Charged states in a finite box: discussion of proposed prescriptions}

Gauss law forbids states with total electric charge different from zero in a box with periodic boundary conditions. In fact the total electric charge is related via the Gauss law to the integral of the divergence of the electric field, which vanishes because of the boundary conditions

$$
Q=\int_{\mathbb{T}_{3}} \mathrm{~d}^{3} x j_{0}(t, \mathbf{x})=\int_{\mathbb{T}_{3}} \mathrm{~d}^{3} x \partial_{k} E_{k}(t, \mathbf{x})=0 .
$$

This is obvious in classical electrodynamics. When temporal-gauge quantization is considered (see e.g. [39]), the above equations is valid at the operatorial level when restricted to the physical Hilbert subspace of gauge-invariant states. The absence of charged states in the physical Hilbert space is an essential obstruction for numerical simulations which aim at calculating properties of charged particles.

A different way to see that charged states do not propagate in finite volume with periodic boundary conditions is the following. Consider QED in a Euclidean box with size $L_{0} \times L_{1} \times L_{2} \times L_{3}$ and periodic boundary conditions for all fields, and with with covariant gauge-fixing,

$$
\begin{aligned}
& S(A, \psi, \bar{\psi})=\int \mathrm{d}^{4} x\left\{\frac{1}{4} F_{\mu \nu}^{2}+\frac{1}{2 \xi}\left(\partial_{\mu} A_{\mu}\right)^{2}+\bar{\psi}\left(\gamma_{\mu} D_{\mu}+m\right) \psi\right\}, \\
& D_{\mu}=\partial_{\mu}+i e A_{\mu} .
\end{aligned}
$$

The action and path-integral measure are invariant under large gauge transformations

$$
\begin{aligned}
& A_{\mu}(x) \rightarrow A_{\mu}(x)-\frac{2 \pi n_{\mu}}{e L_{\mu}}, \\
& \psi(x) \rightarrow e^{2 \pi i\left(L^{-1} n\right)_{\mu} x_{\mu}} \psi(x), \quad \bar{\psi}(x) \rightarrow e^{-2 \pi i\left(L^{-1} n\right)_{\mu} x_{\mu}} \bar{\psi}(x) .
\end{aligned}
$$

Since large gauge transformations are not continuously connected to the identity, they survive any local gauge-fixing procedure. The product $\psi(x) \bar{\psi}(y)$ transforms non-trivially under large gauge transformations if $x \neq y$, therefore the two-point function of charged fields vanishes at distinct points, i.e.

$$
\langle\psi(x) \bar{\psi}(y)\rangle=0, \quad \text { for } x \neq y .
$$

This shows that charged states do not propagate in finite volume with periodic boundary conditions. All prescriptions listed in section 1 solve this problem by destroying large gauge transformations which prevent a non-zero charged two-point function to be defined. The next subsections are devoted to reviewing particular aspects and issues for each prescriptions. 
Before moving to that, I want to add some remarks concerning the expectation values generated by the QED action (2.2), which will be useful later on. I will denote these expectation values simply by $\langle\star\rangle$, defined formally as

$$
\langle P(A, \psi, \bar{\psi})\rangle=\frac{\int[\mathrm{d} A][\mathrm{d} \psi][\mathrm{d} \bar{\psi}] e^{-S(A, \psi, \bar{\psi})} P(A, \psi, \bar{\psi})}{\int[\mathrm{d} A][\mathrm{d} \psi][\mathrm{d} \bar{\psi}] e^{-S(A, \psi, \bar{\psi})}} .
$$

This expression is only formal as both numerator and denominator contain a multiplicative countable infinity due to large gauge transformations, which can be trivially simplified as follows. Decompose the photon field into its constant mode (a.k.a. global zero-mode) plus a fluctuation field

$$
A_{\mu}(x)=e^{-1}\left(L^{-1} \alpha\right)_{\mu}+B_{\mu}(x), \quad \text { with } \quad\left(L^{-1} \alpha\right)_{\mu} \stackrel{\text { def }}{=} L_{\mu}^{-1} \alpha_{\mu} \stackrel{\text { def }}{=} \frac{e}{V} \int \mathrm{d}^{4} x A_{\mu}(x) .
$$

The integration measure over the photon field can be factorized into the integration measure over the constant mode times the integration measure over the fluctuation. Consider an observable that transforms under an irreducible representation of the (Abelian) group of large gauge transformations (2.4), i.e.

$$
P_{z}(A, \psi, \bar{\psi}) \rightarrow P_{z}(A, \psi, \bar{\psi}) \prod_{\mu} z_{\mu}^{n_{\mu}},
$$

for some $z \in \mathbb{C}^{4}$ and any $n \in \mathbb{Z}^{4}$. If $P_{z}$ transforms non-trivially under large gauge transformations, i.e. $z \neq(1,1,1,1)$, then its expectation value vanishes. If $P_{z}$ is invariant under large gauge transformations, then the integration over the constant mode $\alpha$ can be folded into the fundamental domain $(-\pi, \pi)^{4}$. In formulae

$$
\left\langle P_{z}(A, \psi, \bar{\psi})\right\rangle= \begin{cases}\frac{\int_{(-\pi, \pi)^{4}} \mathrm{~d}^{4} \alpha \int[\mathrm{d} B][\mathrm{d} \psi][\mathrm{d} \bar{\psi}] e^{-S(A, \psi, \bar{\psi})} P_{z}(A, \psi, \bar{\psi})}{\int_{(-\pi, \pi)^{4}} \mathrm{~d}^{4} \alpha \int[\mathrm{d} B][\mathrm{d} \psi][\mathrm{d} \bar{\psi}] e^{-S(A, \psi, \bar{\psi})}}, & \text { if } z=(1,1,1,1), \\ 0, & \text { if } z \neq(1,1,1,1),\end{cases}
$$

where it is understood that, up to an immaterial normalization,

$$
[\mathrm{d} B] \stackrel{\text { def }}{=}\left[\prod_{x} \mathrm{~d} B(x)\right] \delta\left(\int \mathrm{d}^{4} x B(x)\right) .
$$

\subsection{Absence of a transfer matrix in $\mathrm{QED}_{\mathrm{TL}}$}

For simplicity we consider periodic boundary conditions for fermions in all directions. $\mathrm{QED}_{\mathrm{TL}}$ is simply defined by constraining the constant mode of the gauge field to zero, i.e.

$$
\langle P(A, \psi, \bar{\psi})\rangle_{\mathrm{TL}} \stackrel{\text { def }}{=} \frac{1}{Z_{\mathrm{TL}}} \int[\mathrm{d} A][\mathrm{d} \psi][\mathrm{d} \bar{\psi}] \delta\left(\int \mathrm{d}^{4} x A(x)\right) e^{-S(A, \psi, \bar{\psi})} P(A, \psi, \bar{\psi}),
$$

where $Z_{\mathrm{TL}}$ is defined in such a way that $\langle 1\rangle_{\mathrm{TL}}=1$. The insertion of a constraint that is non-local in time spoils the transfer-matrix (i.e. Hamiltonian) representation of the path integral.

As noticed by the BMW collaboration in [5], effective masses for charged states defined with the $\mathrm{QED}_{\mathrm{TL}}$ prescription at fixed order in $e^{2}$ diverge in the $T \rightarrow \infty$ limit. Let us be more precise, and consider the two-point function

$$
C(t) \stackrel{\text { def }}{=} \frac{1}{2} \operatorname{tr} \frac{1+\gamma_{0}}{2} \int \mathrm{d}^{3} x\langle\psi(t, \mathbf{x}) \bar{\psi}(0)\rangle_{\mathrm{TL}} .
$$

The polarization projection is not essential and is introduced for the sole purpose to simplify the formulae as much as possible. Explicit calculation (see appendix A in the extended version) of the 
effective mass shows that

$$
m_{\mathrm{eff}}(t) \stackrel{\text { def }}{=}-\frac{\mathrm{d}}{\mathrm{d} t} \ln C(t)=m+\frac{e^{2} T}{16 m L^{3}}+O\left(e^{2} T^{0}\right)+O\left(e^{4}\right) .
$$

Notice that the linear term in $T$ vanishes in the $L \rightarrow \infty$ limit, showing that the sickness of the prescription manifests itself as a non-commutation of limits. Neglecting the fundamental issue that the $\mathrm{QED}_{\mathrm{TL}}$ prescription does not define a local QFT in finite volume, it has been suggested [10] that it is enough to subtract the linear term in $T$ from the effective masses in order to get meaningful results. However notice that, if the $O\left(e^{4}\right)$ terms have a greater degree of divergence in $T$, these might contribute with a non-negligible shift especially in full simulation at unphysically large values of $e$. Whether the higher orders are under control or not is completely unexplored territory.

\subsection{Absence of a transfer matrix in $\mathrm{QED}_{\mathrm{SF}}$}

For simplicity we consider periodic boundary conditions for fermions in all directions. $\mathrm{QED}_{\mathrm{SF}}$ is simply defined by means of

$$
\langle P(A, \psi, \bar{\psi})\rangle_{\mathrm{SF}} \stackrel{\text { def }}{=} \frac{\int_{(-\pi, \pi)^{4}} \mathrm{~d}^{4} \alpha \int[\mathrm{d} B][\mathrm{d} \psi][\mathrm{d} \bar{\psi}] e^{-S(A, \psi, \bar{\psi})} P(A, \psi, \bar{\psi})}{\int_{(-\pi, \pi)^{4}} \mathrm{~d}^{4} \alpha \int[\mathrm{d} B][\mathrm{d} \psi][\mathrm{d} \bar{\psi}] e^{-S(A, \psi, \bar{\psi})}}
$$

for all observables. Notice that eq. (2.14) coincides with eq. (2.9) for observables that are invariant under large gauge transformations. Even though the restriction in the path integral in eq. (2.14) is non local, in the sector that is invariant under large gauge transformations one can use the symmetry to undo the restriction and go back to an expectation value defined in terms of a local action. One might wonder whether the same happens for the two-point function of the charged fermion. Let us see how it works.

Consider the product $\psi(x) \bar{\psi}(0)$, and let us introduce the following non-local function of the photon field

$$
\mathscr{N}_{\mu}(A)=\left\lfloor\frac{\alpha_{\mu}+\pi}{2 \pi}\right\rfloor=\left\lfloor\frac{1}{2}+\frac{e L_{\mu}}{2 \pi V} \int \mathrm{d}^{4} x A_{\mu}(x)\right\rfloor,
$$

where $\lfloor x\rfloor$ denote the floor of $x$, i.e. the largest integer $n$ such that $n \leq x$. Notice that $\mathscr{N}_{\mu}(A)=0$ when inserted in a $\mathrm{QED}_{\mathrm{SF}}$ expectation value because of the restriction $-\pi<\alpha_{\mu}<\pi$, therefore

$$
\langle\psi(x) \bar{\psi}(0)\rangle_{\mathrm{SF}}=\left\langle e^{2 \pi i\left(L^{-1} x\right)_{\mu} \mathscr{N}_{\mu}(A)} \psi(x) \bar{\psi}(0)\right\rangle_{\mathrm{SF}} .
$$

It is easy to check that, under a large gauge transformation (2.4),

$$
\mathscr{N}_{\mu}(A) \rightarrow \mathscr{N}_{\mu}(A)-n_{\mu},
$$

and the observable $e^{2 \pi i\left(L^{-1} x\right)_{\mu} \mathscr{N}_{\mu}(A)} \psi(x) \bar{\psi}(0)$ is invariant. Since the $\mathrm{QED}_{\mathrm{SF}}$ expectation value coincides with the unconstrained one for observables that are invariant under large gauge transformations, one concludes that

$$
\langle\psi(x) \bar{\psi}(0)\rangle_{\mathrm{SF}}=\left\langle e^{2 \pi i\left(L^{-1} x\right)_{\mu} \mathscr{N}_{\mu}(A)} \psi(x) \bar{\psi}(0)\right\rangle .
$$

Notice that eq. (2.18) shifts the non-locality from the path-integral measure (in the $\mathrm{QED}_{\mathrm{SF}}$ expectation value) to the observable (in the QED expectation value). In particular a Hamiltonian representation for the two-point function seems not to be possible as, in the formulation with a local path-integral measure, the observable is non-local in time, moreover the non-locality range is equal to the whole lattice size. ${ }^{1}$

\footnotetext{
${ }^{1}$ This is by no means a proof that a Hamiltonian representation does not exist. In principle locality might be hidden by an inconvenient choice of fundamental variables, and made manifest by a smart change of variables. I have been privately
} 


\section{3 (Non)renormalization of composite operators and $Q_{E} D_{L}$}

The $\mathrm{QED}_{\mathrm{L}}$ prescription is defined by replacing the photon-field integration measure with

$$
[\mathrm{d} A] \rightarrow[\mathrm{d} A]\left[\prod_{t}\right] \prod_{\mu=0}^{4} \delta\left(\int \mathrm{d}^{3} x A_{\mu}(t, \mathbf{x})\right) .
$$

This constraint is local in time, therefore $\mathrm{QED}_{\mathrm{L}}$ admits a Hamiltonian. However this Hamiltonian is non-local in space. Locality is a core property of quantum field theory, and several properties that we usually give for granted depend on locality, such as renormalizability, operator product expansion, effective-field theory description of low-energy modes. As we will see, the renormalization of composite operators is broken by the constraint defining $\mathrm{QED}_{\mathrm{L}}$. Before moving on, I want to comment on a couple of myths existing around $\mathrm{QED}_{\mathrm{L}}$.

- Since we are subtracting low-modes, i.e. IR modes, these should not affect the UV properties of the theory. This is misleading on several levels. First one should notice that any notion of IR/UV decoupling (which is deeply related to the concept of effective field theory) makes sense only in a local theory. Second one should notice that the constraint in eq. (2.19) eliminates Fourier components with $p=\left(p_{0}, \boldsymbol{0}\right)$ for any value of $p_{0}$ admitted by the boundary conditions. Therefore the constraint in eq. (2.19) eliminates momenta with arbitrarily large norm.

- The $Q E D_{L}$ prescription is equivalent to coupling $Q E D$ to a classical uniform charge density. Let $\mathscr{J}_{\mu}(x)$ be a classical electromagnetic current. The action of QED coupled to the classical current is

$$
S_{\mathscr{J}}=S_{0}+i e \int \mathrm{d}^{4} x A_{\mu}(x) \mathscr{J}_{\mu}(x) .
$$

The dynamics of the quantum fields are determined by the probability distribution that defines the path-integral, while the dynamics of the classical current is determined by classical equations of motion. In particular the classical current should not be integrated over. The dynamics of the quantum fields in presence of a given classical current is local. $\mathrm{QED}_{\mathrm{L}}$ is obtained by choosing $\mathscr{J}_{\mu}(x)$ to be invariant under spatial translations, and by integrating over it in the path integral. The integration spoils the interpretation of $\mathscr{J}_{\mu}(x)$ as a classical current.

Let us discuss the renormalization of composite operators. For sake of presentation, in place of $\mathrm{QED}_{\mathrm{L}}$, we shall consider the $\phi^{4}$ theory on $\mathbb{R} \times T_{3}$ where $T_{3}$ is the maximally symmetric three-torus with linear size equal to $L$. We write the action having in mind the renormalized perturbative expansion

$$
S=\int_{L^{3}} \mathrm{~d}^{4} x\left\{\frac{1}{2} \phi\left(-\square+m^{2}\right) \phi+\frac{\lambda}{4 !} \phi^{4}+\frac{\delta Z}{2} \phi\left(-\square+m^{2}\right) \phi+\frac{\delta m^{2}}{2} \phi^{2}+\frac{\delta \lambda}{4 !} \phi^{4}\right\}
$$

where $\phi$ is the renormalized field, $m$ and $\lambda$ are renormalized parameters, while $\delta Z, \delta m^{2}$ and $\delta \lambda$ are the coefficients of the counterterms that are supposed to be at least $O(\lambda)$. We impose a constraint on the path-integral measure that mimics $\mathrm{QED}_{\mathrm{L}}$

$$
[\mathrm{d} \phi] \rightarrow[\mathrm{d} \phi]\left[\prod_{t}\right] \delta\left(\int \mathrm{d}^{3} x \phi(t, \mathbf{x})\right) .
$$

The propagator $\Delta^{\prime}(x)$ in coordinate space can be split in two pieces

$$
\Delta^{\prime}(x)=\Delta(x)-\Delta_{0}\left(x_{0}\right)
$$

informed by Schierholz that he is working on a proof that a Hamiltonian representation of the two-point function actually exists, and this would be an extremely interesting result. 
the propagator in the full theory without constraint

$$
\Delta(x)=\frac{1}{L^{3}} \sum_{\mathbf{p} \in \frac{2 \pi}{L} \mathbb{Z}^{3}} \int \frac{\mathrm{d} p_{0}}{2 \pi} \frac{e^{i p x} e^{-\Lambda^{-2}\left(p^{2}+m^{2}\right)}}{p^{2}+m^{2}},
$$

and the subtraction

$$
\Delta_{0}\left(x_{0}\right)=\frac{1}{L^{3}} \int \frac{\mathrm{d} p_{0}}{2 \pi} \frac{e^{i p_{0} x_{0}} e^{-\Lambda^{-2}\left(p_{0}^{2}+m^{2}\right)}}{p_{0}^{2}+m^{2}},
$$

where the heat-kernel regularization has been used. The counterterms $\delta Z$ and $\delta m$ are determined (up to a finite part) by requiring that the two-point function $\langle\phi(x) \phi(0)\rangle$ is finite in the $\Lambda \rightarrow \infty$ limit so long as $x \neq 0$. Analogously $\delta \lambda$ is determined by requiring that the four-point function of the renormalized fields is finite. The calculation of the action counterterms is standard, as the constraint turns out to play no role in the identification of the divergences of two and four-point functions at one loop. We give the results

$$
\delta Z=O\left(\lambda^{2}\right), \quad \delta m^{2}=-\frac{\lambda \Lambda^{2}}{2(4 \pi)^{2}}+\frac{\lambda m^{2}}{(4 \pi)^{2}} \ln \frac{\Lambda}{\mu}+O\left(\lambda^{2}\right), \quad \delta \lambda=\frac{3 \lambda^{2}}{(4 \pi)^{2}} \ln \frac{\Lambda}{\mu}+O\left(\lambda^{3}\right) .
$$

As a consequence of the constraint in eq. (2.22), the propagator $\Delta^{\prime}(x)$ is not a smooth function for $x \neq 0$. In fact a little algebra shows that

$$
\square \Delta^{\prime}(0, \mathbf{x})=-\frac{\Lambda}{(4 \pi)^{1 / 2} L^{3}}+O\left(\Lambda^{0}\right)
$$

for any $\mathbf{x} \neq \mathbf{0}$. This non-local divergence will propagate into loops by generating unwanted mixing with non-local operators. Let us see how in a particular example, which has been cooked up to show this feature in a very simple diagram.

We consider the bare operator $(\square \phi)^{2}$. In a local theory, the divergences generated by the insertion of $(\square \phi)^{2}(x)$ in general expectation values can be subtracted by inserting local counterterms in $x$. These counterterms are all possible local operators with the same quantum numbers as $(\square \phi)^{2}$, and with dimension not greater than 6 . In other terms, a renormalized operator can be defined as follows

$$
\left[(\square \phi)^{2}\right]_{R}(x)=(\square \phi)^{2}(x)+\sum_{d_{\mathscr{O}} \leq 6} c_{\alpha} \Lambda^{6-d_{\mathscr{O}}} \mathscr{O}(x)+c_{\mathrm{id}} \Lambda^{6},
$$

where the $c_{\alpha}$ 's are $O(\lambda)$ coefficients which diverge at most logarithmically ( $c_{\mathrm{id}}$ is the so called mixing with the identity). The renormalized operator is defined such that all expectation values of the form

$$
\left\langle\phi\left(z^{1}\right) \cdots \phi\left(z^{n}\right)\left[(\square \phi)^{2}\right]_{R}(x)\right\rangle
$$

are finite in the $\Lambda \rightarrow \infty$ limit so long as all point are pairwise distinct. We will show explicitly that in the theory with constraint (2.22), it is not possible to choose local counterterms as in eq. (2.28) so that the following expectation value is finite

$$
\left\langle\phi\left(z^{1}\right) \phi\left(z^{2}\right)\left[(\square \phi)^{2}\right]_{R}(x)\right\rangle_{c} .
$$

The connected expectation value is taken so to kill the mixing with the identity. We can calculate

$$
\begin{array}{r}
\left\langle\phi\left(z^{1}\right) \phi\left(z^{2}\right)(\square \phi)^{2}(x)\right\rangle_{c}=2\left\langle\phi\left(z^{1}\right) \square \phi(x)\right\rangle\left\langle\phi\left(z^{2}\right) \square \phi(x)\right\rangle+ \\
-\lambda \int \mathrm{d}^{4} y \Delta^{\prime}\left(z^{1}-y\right) \Delta^{\prime}\left(z^{2}-y\right)\left[\square \Delta^{\prime}(x-y)\right]^{2}+O\left(\lambda^{2}\right) .
\end{array}
$$

The two-point functions in the r.h.s. have the same divergence as in eq. (2.27) when $z_{0}^{1}=x_{0}$ for all values of $\mathbf{z}^{1}$, or $z_{0}^{2}=x_{0}$ for all values of $\mathbf{z}^{2}$. This divergence is obviously non-local. Even when this divergence is avoided, the loop integral has extra divergences with respect to the infinite-volume case. 
The full analysis of divergences is quite tedious but straightforward. One can expand the propagators $\Delta^{\prime}(x-y)$ inside the loop by means of eq. (2.23). It is interesting to look at one particular contribution, i.e. the one that is obtained by replacing both loop propagators by $-\Delta_{0}\left(x_{0}-y_{0}\right)$. Some calculation yields

$$
\begin{aligned}
& -\lambda \int \mathrm{d}^{4} y \Delta^{\prime}\left(z^{1}-y\right) \Delta^{\prime}\left(z^{2}-y\right)\left[\partial_{0}^{2} \Delta_{0}\left(x_{0}-y_{0}\right)\right]^{2}= \\
& =-\frac{\lambda}{(8 \pi)^{1 / 2} L^{6}} \Lambda \int \mathrm{d}^{4} y \Delta^{\prime}\left(z^{1}-y\right) \Delta^{\prime}\left(z^{2}-y\right) \delta\left(y_{0}-x_{0}\right)+O\left(\Lambda^{0}\right) .
\end{aligned}
$$

The appearance of this divergence is fairly simple to understand. If we set $\Lambda=\infty$ then by definition,

$$
\partial_{0}^{2} \Delta_{0}\left(x_{0}-y_{0}\right)=-\delta\left(x_{0}-y_{0}\right)+m^{2} \Delta_{0}\left(x_{0}-y_{0}\right),
$$

and when we square this expression, we get a $\left[\delta\left(x_{0}-y_{0}\right)\right]^{2}$ which is a linear divergence, whose exact value can be calculated only at finite regulator. The divergence in eq. (2.32) would be canceled by the insertion in the r.h.s. of eq. (2.28) of the following operator

$$
\frac{\lambda}{2(8 \pi)^{1 / 2} L^{6}} \Lambda \int \mathrm{d}^{3} \mathbf{y} \phi^{2}\left(x_{0}, \mathbf{y}\right),
$$

which is obviously non local. Again the effect of non-locality shows as a non-commutation of limits. If we take the infinite volume limit before the $\Lambda \rightarrow \infty$ limit, this contribution vanishes.

Even though renormalization at one loop of operators with dimension not greater than 4 seems to happen in the same way as in infinite volume, I have shown that renormalization by local counterterms breaks down even at one loop for operators with high enough dimension. This will obviously propagate into the Symanzik expansion of low dimensional operators (and in particular of the action) with unexplored consequences.

Translating the above example to $\mathrm{QED}_{\mathrm{L}}$ is not trivial, as the photon propagator cannot be decomposed in a simple way as in eq. (2.23). Also one may need to go to higher order to reproduce an analogous mechanism. A full analysis of the finite-volume divergences in $\mathrm{QED}_{\mathrm{L}}$ is nowhere in sight, and I hope that I have convinced the reader that this is not a trivial matter. The above analysis is maximally relevant for strategies that rely on unphysically large values of $\alpha_{\mathrm{em}}$ as higher-loop effects are amplified, and on simultaneous extrapolations to $L \rightarrow \infty$ and $a \rightarrow 0$ as these two limits do not commute. Precision can not be claimed without having the effects of non-locality under control.

\subsection{A local formulation: $\mathrm{QED}_{\mathrm{m}}$}

$\mathrm{QED}_{\mathrm{m}}$ is defined by giving a mass to the photon, i.e. by replacing the action with

$$
S_{\mathrm{m}}(A, \psi, \bar{\psi}) \stackrel{\text { def }}{=} S(A, \psi, \bar{\psi})+\frac{m_{\gamma}^{2}}{2} \int \mathrm{d}^{4} x A_{\mu}^{2}
$$

which gives a consistent (in particular, local) QFT in finite volume, defined order by order in the perturbative expansion. The mass term introduces a soft breaking of gauge symmetry (like the gaugefixing term). The renormalization of $\mathrm{QED}_{\mathrm{m}}$ is well understood. The $m_{\gamma} \rightarrow 0$ limit in infinite volume is widely studied in the literature. However $\mathrm{QED}_{\mathrm{m}}$ employs two IR regulators: the photon mass and the finite volume. The $L \rightarrow \infty$ and $m_{\gamma} \rightarrow 0$ limits do not commute, and infinite-volume QED is recovered only if the $L \rightarrow \infty$ limit is taken before the $m_{\gamma} \rightarrow 0$ limit. Non-commutation of limits makes usually the extrapolation particularly challenging. However it has to be noted that, as long as $m_{\gamma} \neq 0$, the infinite-volume limit is reached easily as the finite-volume corrections are exponentially small (e.g. for masses of stable states). I want to investigate here a bit more in detail the implications of the non-commutation of limits. 
The lengthy calculation in appendix $\mathrm{C}$ in the extended version shows that the fermion two-point function in $\mathrm{QED}_{\mathrm{m}}$ can be represented as follows

$$
\left\langle\psi(x) \bar{\psi}\left(0^{+}\right)\right\rangle_{\mathrm{m}}=\frac{\sum_{q \in \mathbb{Z}^{4}} e^{-\frac{e^{2}}{2 m_{\gamma}^{2} V}(L q+x)_{\mu}^{2}}\left\langle\psi(x) \bar{\psi}\left(0^{+}\right) e^{-\frac{m_{\gamma}^{2}}{2} \int \mathrm{d}^{4} z B_{\mu}^{2}(z)} \delta_{Q, q}\right\rangle_{\mathrm{TL}}}{\sum_{q \in \mathbb{Z}^{4}} e^{-\frac{e^{2}}{2 m_{\gamma} V}(L q)_{\mu}^{2}}\left\langle e^{-\frac{m_{\gamma}^{2}}{2} \int \mathrm{d}^{4} z B_{\mu}^{2}(z)} \delta_{Q, q}\right\rangle_{\mathrm{TL}}}
$$

where $Q_{\mu}$ is the electric charge (in units of $e$ ) operator defined by interpreting $\mu$ as temporal direction and localized on the time-slice $x_{\mu}=0$, i.e.

$$
Q_{\mu}=\int \mathrm{d}^{4} z \delta\left(z_{\mu}\right) \bar{\psi} \gamma_{\mu} \psi(z)
$$

We want to study the $m_{\gamma} \rightarrow 0$ limit at fixed volume of the two-point function in time momentum representation

$$
C(t, \mathbf{p}) \stackrel{\text { def }}{=} \int_{L_{1} \times L_{2} \times L_{3}} \mathrm{~d}^{3} x e^{-i \mathbf{p x}}\left\langle\psi(t, \mathbf{x}) \bar{\psi}\left(0^{+}\right)\right\rangle_{\mathrm{m}} .
$$

In the $m_{\gamma} \rightarrow 0$ limit, the denominator of eq. (2.36) is dominated by the term $q=0$, i.e.

$$
\sum_{q \in \mathbb{Z}^{4}} e^{-\frac{e^{2}}{2 m_{\gamma}^{2} V}(L q)_{\mu}^{2}}\left\langle e^{-\frac{m_{\gamma}^{2}}{2} \int \mathrm{d}^{4} z B_{\mu}^{2}(z)} \delta_{Q, q}\right\rangle_{\mathrm{TL}} \stackrel{m_{\gamma} \rightarrow 0}{=}\left\langle\delta_{Q, 0}\right\rangle_{\mathrm{TL}}+O\left(m_{\gamma}^{2}\right) .
$$

When the numerator of eq. (2.36) is plugged into eq. (2.38), the $m_{\gamma} \rightarrow 0$ limit is obtained by saddle point. For $0<t<L_{0} / 2$ the saddle point is located at $q=0$ and $\mathbf{x}=0$, while for $L_{0} / 2<t<L_{0}$ the saddle point is located at $q=(-1, \mathbf{0})$ and $\mathbf{x}=0$. Let us consider only the $0<t<L_{0} / 2$ case for simplicity, i.e.

$$
C(t, \mathbf{p}) \stackrel{m_{\gamma} \rightarrow 0}{=} \frac{\left(2 \pi e^{-2} m_{\gamma}^{2} V\right)^{3 / 2}}{\left\langle\delta_{Q, 0}\right\rangle_{\mathrm{TL}}} e^{-\frac{e^{2}}{2 m_{\gamma} V} t^{2}}\left\langle\psi(t, \mathbf{0}) \bar{\psi}\left(0^{+}\right) \delta_{Q, 0}\right\rangle_{\mathrm{TL}}\left\{1+O\left(m_{\gamma}^{2}\right)\right\} .
$$

The two-point function vanishes exponentially in the $m_{\gamma} \rightarrow 0$ limit for $t \neq 0$ as it should, since large gauge transformations become symmetries in the $m_{\gamma} \rightarrow 0$ limit. Moreover the leading term in the $m_{\gamma} \rightarrow 0$ limit does not depend on the momentum $\mathbf{p}$, as a direct consequence of the fact that the integral is dominated by the value of the integrand at $\mathbf{x}=\mathbf{0}$. At this order, the effective mass is

$$
m_{\mathrm{eff}}(t, \mathbf{p}) \stackrel{\text { def }}{=}-\frac{\mathrm{d}}{\mathrm{d} t} \ln C(t, \mathbf{p})=\frac{e^{2}}{m_{\gamma}^{2} V} t-\frac{\mathrm{d}}{\mathrm{d} t} \ln \left\langle\psi(t, \mathbf{0}) \bar{\psi}\left(0^{+}\right) \delta_{Q, 0}\right\rangle_{\mathrm{TL}}+O\left(m_{\gamma}^{2}\right) .
$$

The term proportional to $t$ in the effective mass is the one identified in [35]. Notice that this term vanishes in the infinite-volume limit and diverges in the $m_{\gamma} \rightarrow 0$ limit. Even if this term is removed by hand, the non-commutation of limits is not resolved. If $m_{\gamma}$ is too small, the physical dependence of the effective mass on the momentum is suppressed, and effectively one is just using a complicated method to extract the $\mathrm{QED}_{\mathrm{TL}}$ effective mass in coordinate space. In this regime it would be impossible to construct states with definite momentum, which are necessary e.g. in the calculation of transition amplitudes. It is worth noticing that the term proportional to $t$ is large in the exploratory study in [35], which suggests in fact that this study is performed in the regime in which eq. (2.41) is valid. A possible way to verify or disprove this is to calculate the effective mass at different values of $\mathbf{p}$ and check whether it is constant or not.

\subsection{A local formulation: QED $_{\mathrm{C}}$}

$\mathrm{QED}_{\mathrm{C}}$ is defined by means of $\mathrm{C}^{\star}$ boundary conditions (a.k.a. C-parity boundary conditions) along the 
spatial directions for all fields,

$$
A_{\mu}\left(x+L_{k} \hat{k}\right)=-A_{\mu}(x), \quad \psi\left(x+L_{k} \hat{k}\right)=C^{-1} \bar{\psi}^{T}(x),
$$

where $C$ is the charge-conjugation matrix for spinors. $\mathrm{QED}_{\mathrm{C}}$ is a consistent (in particular, local) QFT at finite volume, defined order by order in the perturbative expansion. Some features and delicate aspects of $\mathrm{QED}_{\mathrm{C}}$ are listed in the following.

- The total electric charge is not constrained to be zero by the Gauss law

$$
Q=\int_{\mathbb{T}_{3}} \mathrm{~d}^{3} x j_{0}(t, \mathbf{x})=\int_{\mathbb{T}_{3}} \mathrm{~d}^{3} x \partial_{k} E_{k}(t, \mathbf{x})=-2 \sum_{k} \int \mathrm{d}^{3} x \delta\left(x_{k}\right) E_{k}(t, \mathbf{x}),
$$

since the electric field is antiperiodic in the spatial directions. In other works, the electric flux produce by a particle can escape the torus and be eaten by the image particle (which has opposite charge).

- Large gauge transformations of the form (2.4) do not leave the photon field boundary conditions inveriant and are therefore not a symmetry of the theory.

- Charge conservation and, similarly, flavor conservation are partially broken by the boundary conditions. The electric charge $Q$ is not conserved, but the quantum number $(-1)^{Q}$ is. The same happens for each individual flavor number. This is enough to protect all stable mesons and most of the stable baryons from unphysical decays. Some baryons, that would be stable in infinite volume, mix with lighter ones because of the boundary conditions (e.g. $\Xi^{-}$mixes with $p$ ). In effective masses this effect appears (again!) as a non-commutation of IR limit

$$
\begin{aligned}
& m_{\mathrm{eff}}(t \mid L)=-\lim _{T \rightarrow \infty} \frac{\mathrm{d}}{\mathrm{d} t} \ln \int \mathrm{d}^{3} x\left\langle\Xi_{-}^{\dagger}(t, \mathbf{x}) \Xi_{-}(0, \mathbf{x})\right\rangle \stackrel{\text { def }}{=}-\frac{\mathrm{d}}{\mathrm{d} t} \ln C(t \mid L), \\
& \lim _{t \rightarrow \infty} \lim _{L \rightarrow \infty} m_{\text {eff }}(t \mid L)=M_{\Xi^{-}}, \quad \lim _{L \rightarrow \infty} \lim _{t \rightarrow \infty} m_{\mathrm{eff}}(t \mid L)=M_{p} .
\end{aligned}
$$

These spurious mixing are due to flavored mesons traveling around the torus and are suppressed exponentially with the volume, more precisely the following decomposition for the correlator $C(t \mid L)$ exists

$$
C(t \mid L)=C_{0}(t \mid L)+e^{-\mu L} C_{1}(t \mid L),
$$

where both $C_{0}(t \mid L)$ and $C_{1}(t \mid L)$ are finite in the $L \rightarrow \infty$ limit at fixed $t$, and also

$$
-\lim _{t \rightarrow \infty} \frac{\mathrm{d}}{\mathrm{d} t} \ln C_{0}(t \mid L)=M_{\Xi^{-}}(L), \quad-\lim _{t \rightarrow \infty} \frac{\mathrm{d}}{\mathrm{d} t} \ln C_{1}(t \mid L)=M_{p}(L) .
$$

The suppression exponent $e^{-\mu L}$ is calculated to be $O\left(10^{-10}\right)$ at physical quark masses and $M_{\pi} L=4$.

- The partial breaking of flavor symmetry does not affect the renormalization of composite operators. Thanks to locality, the divergent part of the renormalization constants does not depend on the volume.

- The non-universal finite volume corrections to the hadron masses vanish as $L^{-4}$ (as opposed to $L^{-3}$ in $\mathrm{QED}_{\mathrm{L}}$ ).

- A gauge-invariant representation for the charged-state interpolating operators is possible. 


\section{IR divergences and decay rates}

In this section I will briefly discuss radiative corrections to decay rates. Subsection 3.1 is completely textbook, and contains a review of some basic facts about IR soft divergences. I have chosen to include this subsection since IR divergences do not belong to the standard toolbox of the lattice community. Subsection 3.2 is a review of the exploratory calculation of the pion and kaon decay rate at $O\left(\alpha_{\mathrm{em}}\right)$ presented at this conference by the RM-SOTON collaboration. This subsections gives me the chance also to discuss the separation of radiative corrections in a universal piece (a.k.a. innerbremsstrahlung) and a structure-dependent piece, which is a useful tool for numerical simulations, and it is also fundamental in order to understand the jargon of the experimentalist and the phenomenologist. Finally in subsection 3.3 I argue that radiative corrections can be enhanced by large logarithms due to quasi-collinear divergences in decay rates of heavy mesons. This phenomenology is completely non-perturbative and hinders the reliability of phenomenological estimates of radiative corrections. It is where our community may be able to give an important contribution hopefully in the near future.

\subsection{Some facts about soft divergences}

If one wants to extract S-matrix elements from numerical simulations when QCD is coupled to QED, besides dealing with the standard complications due to the Euclidean setup and the finite volume, one has to deal also with IR divergences. We assume that all charged asymptotic particles are massive. In this case IR (soft) divergences are due to soft photons (i.e. photons with four-momentum $k$, such that $k^{2}$ is asymptotically small) in loops and in the final state.

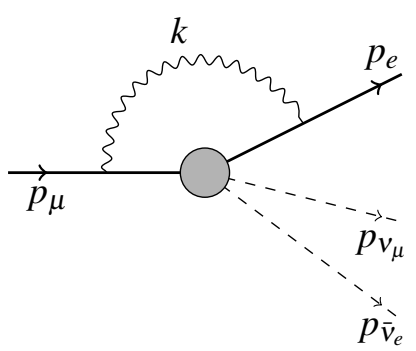

Figure 1: Feynman diagram that contributes to the $\mu^{-} \rightarrow e^{-}+\bar{v}_{e}+v_{\mu}$ amplitude at order $\alpha_{\mathrm{em}}$. The gray circle represents the insertion of the EW effective Hamiltonian. The loop integral is IR divergent.

Let us review briefly how soft divergences are generated in loops. Roughly speaking they are due to the fact that the photon propagator $1 / k^{2}$ is singular when $k^{2} \rightarrow 0$. However it has to be noted that this singularity is integrable, hence generally not enough to produce a divergence in the Feynman integrals. In fact $n$-point functions in coordinate space (both in Minkowskian and Euclidean spacetime) are IR finite. Soft divergences appear in 1PI diagrams when external momenta are chosen on the mass shell. In order to illustrate how this happens, let us consider the EW decay $\mu^{-} \rightarrow e^{-}+\bar{v}_{e}+v_{\mu}$. For simplicity we can work with Fermi's effective theory (i.e. in the $M_{W} \rightarrow \infty$ limit), and consider the one-loop 1PI diagram in figure 3.1. The S-matrix element is calculated by setting the external momenta on the mass-shell, i.e. $p_{\mu}^{2}=-m_{\mu}^{2}$ and $p_{e}^{2}=-m_{e}^{2}$ (in Euclidean notation). If $k$ is the photon momentum, the charged-particle propagators inside the loop are

$$
\frac{-i\left(\not p_{\star}+\not k\right)+m_{\star}}{\left(p_{\star}+k\right)^{2}+m_{\star}^{2}}=\frac{-i\left(\not p_{\star}+\not k\right)+m_{\star}}{2 p_{\star} k+k^{2}}=\frac{-i \not p_{\star}+m_{\star}}{2 p_{\star} k}+O\left(k^{0}\right) .
$$


In the $k \rightarrow 0$ limit the charged propagators go on-shell and contribute with a $k^{-1}$ singularity to the loop integrand. Since there is two of them, and a photon propagator, the loop integral restricted to the soft region is proportional to

$$
\int_{k^{2}<\Lambda} \frac{\mathrm{d}^{4} k}{(2 \pi)^{4}} \frac{1}{2 p_{\mu} k} \frac{1}{2 p_{e} k} \frac{1}{k^{2}},
$$

which is logarithmically divergent. Notice that only one singular matter propagator would not be enough to generate the divergence.

At fixed order in $\alpha_{\mathrm{em}}$ transition amplitudes are IR divergent because of loops of soft photons. Bloch and Nordsieck [40] pointed out that these divergences cancel in decay rates, when integrated over an arbitrary number of soft photons in the final state. Let us review briefly the physics significance of the Bloch-Nordsieck cancellation mechanism and how it works. Let us consider a generic process $\alpha \rightarrow \beta$ involving any number of hard particles of any kind. Let $\mathbf{p}_{n}$ be the momentum of the $n$-th particle in this process. Let us consider also the process $\alpha \rightarrow \beta+N \gamma$ in which the final state contains also $N$ soft photons, with the following properties

1. the $n$-th incoming particle has momentum $\mathbf{p}_{n}^{\prime}=\mathbf{p}_{n}$;

2. the $n$-th outgoing hard particle has momentum $\mathbf{p}_{n}^{\prime}$, with $\left|\mathbf{p}_{n}^{\prime}-\mathbf{p}_{n}\right|<\Delta p$;

3. each soft outgoing photon has energy less than $\Delta E$.

If $\Delta E$ and $\Delta p$ are the energy and momentum resolution of the detector, then the two processes are experimentally indistinguishable. The probability rate to transition from $\alpha$ to $\beta$ turns out to be infinite at any order in $\alpha_{\mathrm{em}}$ beyond tree-level. However this probability rate is not relevant from the experimental point of view. In fact only the probability rate to transition from $\alpha$ to any final state that is experimentally indistinguishable from $\beta$ is relevant. It turns out that the latter probability rate is IR finite at any order in the perturbative expansion.

Let us try to be a bit more precise. ${ }^{2}$ Let us introduce an IR regulator, e.g. a mass $m_{\gamma}$ for the photon. Let $M_{\alpha \beta}\left(\mathbf{k}_{1}, \ldots, \mathbf{k}_{N} ; m_{\gamma}\right)$ be the transition amplitude for the process $\alpha \rightarrow \beta+N \gamma$, where $\mathbf{k}_{r}$ is the momentum the $r$-th soft photon. Notice that the soft photons cannot carry a large amount of total energy away (even if $N$ is large and $m_{\gamma}=0$ ), otherwise by energy conservation the energy of some of the outgoing particles would be notably affected. Therefore it makes sense to consider the transition rate summed over any number of outgoing soft photons with individual energy less than $\Delta E$ and total energy less then $E_{T}=O(\Delta p)$, i.e.

$$
\begin{aligned}
& \Gamma_{\alpha \beta}(\left.\Delta E, E_{T} ; m_{\gamma}\right)= \\
& \quad=\mathscr{N}_{\alpha} \sum_{N=0}^{\infty} \frac{1}{N !} \int_{\substack{\left|\mathbf{k}_{r}\right|<\Delta E \\
\sum_{r=1}^{N}\left|\mathbf{k}_{r}\right|<E_{T}}}\left\{\prod_{r=1}^{N} \frac{\mathrm{d}^{3} k_{r}}{(2 \pi)^{3} 2\left(m_{\gamma}^{2}+\mathbf{k}^{2}\right)^{1 / 2}}\right\}\left|M_{\alpha \beta}\left(\mathbf{k}_{1}, \ldots, \mathbf{k}_{N} ; m_{\gamma}\right)\right|^{2},
\end{aligned}
$$

where $\mathscr{N}_{\alpha}$ is a normalization factor depending only on the initial state, and the sum over the polarizations of the soft photons is understood. The nontrivial fact about soft divergences is that the asymptotic behaviour of the transition amplitude in the $m_{\gamma} \rightarrow 0$ and $\mathbf{k} \rightarrow 0$ limit is calculable at all orders in the perturbative expansion

$$
M_{\beta \alpha}\left(\mathbf{k}_{1}, \ldots, \mathbf{k}_{N} ; m_{\gamma}\right) \stackrel{\mathbf{k} \sim m_{\gamma} \rightarrow 0}{\simeq} \hat{M}_{\beta \alpha}(\mu)\left(\frac{m_{\gamma}}{\mu}\right)^{\frac{\alpha_{\mathrm{em}}}{2} A_{\beta \alpha}} \prod_{r=1}^{N} \sum_{n} \frac{e q_{n} \eta_{n} p_{n} \cdot \varepsilon_{r}^{*}}{p_{n} \cdot k_{r}} .
$$

\footnotetext{
${ }^{2}$ The upcoming discussion is textbook (see e.g. section 13.4 in [41]). The concepts of factorization, exponentiation and universality of soft divergences have their root in the fundamental work of Yennie, Frautschi, Suura [42], and Grammer, Yennie [43], and have been beautifully summarized by Weinberg [44]. The interpretation of IR divergences as break-down of the perturbative expansion of transition amplitudes has been analyzed in detail by Lee and Nauenberg [45].
} 
Here $p_{n}, m_{n}$, and $q_{n}$ are the four-momentum, mass and electric charge (in units of $e$ ) of the $n$-th hard particle, while $\eta_{n}$ is defined to be equal to +1 and -1 for particles in the final and initial state respectively. $k_{r}$ and $\varepsilon_{r}$ are the four-momentum and polarization four-vector of the $r$-th soft photon. $\mu$ is an arbitrary energy scale introduced to the sole purpose to match the dimensions, $\hat{M}_{\beta \alpha}(\mu)$ does not depend on $m_{\gamma}$. Finally $A_{\beta \alpha}$ is a known exponent

$$
\begin{aligned}
& A_{\beta \alpha} \stackrel{\text { def }}{=}-\frac{1}{2 \pi} \sum_{n m} \frac{q_{n} q_{m} \eta_{n} \eta_{m}}{\beta_{n m}} \ln \frac{1+\beta_{n m}}{1-\beta_{n m}}+i \sum_{n \neq m} \frac{q_{n} q_{m}}{\beta_{n m}} \delta_{\eta_{n}, \eta_{m}}, \\
& \beta_{n m} \stackrel{\text { def }}{=} \sqrt{1-\frac{m_{n}^{2} m_{m}^{2}}{\left(p_{n} \cdot p_{m}\right)^{2}}} .
\end{aligned}
$$

It is usually said that soft divergences factorize, since the virtual soft photons contribute to the transition amplitude with the factor $\left(m_{\gamma} / \mu\right)^{\frac{\alpha_{\mathrm{em}}}{2} A_{\beta \alpha}}$, and each real soft photon contributes with a factor $\sum_{n} e q_{n} \eta_{n}\left(p_{n} \cdot \varepsilon_{r}^{*}\right) /\left(p_{n} \cdot k_{r}\right)$. It is also usually said that soft divergences are universal in the sense that these factors are completely determined by masses, charges and momenta of the particles in the initial and final states. In particular the factors depend neither on the microscopic interactions that produce the scattering process nor on the spin and internal structure of the particles.

Notice that the virtual-photon factor gives contributions to all orders in the perturbative expansion. The real part of $A_{\alpha \beta}$ is proven to be always positive, therefore the formally resummed transition amplitude vanishes when the IR regulator is removed while the momenta of the soft photons are kept small but non-vanishing. This phenomenon is known as evaporation of the $S$-matrix. ${ }^{3}$ If $\Delta E, E_{T}$ and $m_{\gamma}$ are small enough, one can plug the approximation (3.4) into the formula (3.3) for the integrated transition rate. Because of the singularity at $\mathbf{k}_{r} \rightarrow 0$, the phase-space integral in eq. (3.3) diverges when the IR regulator is removed. This divergence compensates exactly the vanishing factor due to virtual soft photons, so that the transition probability rate becomes finite and non-zero in the $m_{\gamma} \rightarrow 0$ limit,

$$
\begin{aligned}
& \Gamma_{\alpha \beta}\left(\Delta E, E_{T}\right)=\lim _{m_{\gamma} \rightarrow 0} \Gamma_{\alpha \beta}\left(\Delta E, E_{T} ; m_{\gamma}\right) \stackrel{\Delta E \sim E_{T} \rightarrow 0}{\simeq} \\
& \simeq \mathscr{N}_{\alpha} F\left(\frac{\Delta E}{E_{T}}, \operatorname{Re} A_{\beta \alpha}\right)\left(\frac{\Delta E}{\mu}\right)^{\alpha_{\mathrm{em}} \operatorname{Re} A_{\beta \alpha}}\left|\hat{M}_{\beta \alpha}(\mu)\right|^{2},
\end{aligned}
$$

where $F(x, A)$ is a known kinematic function which is reported for completeness

$$
F(x, A)=\frac{1}{\pi} \int_{-\infty}^{\infty} \mathrm{d} u \frac{\sin u}{u} \exp \left(\alpha_{\mathrm{em}} A \int_{0}^{x} \mathrm{~d} \omega \frac{e^{i \omega u}-1}{\omega}\right) .
$$

Notice that the transition rate in the resummed form (3.8) is finite for any value of $\Delta E$ and vanishes in

\footnotetext{
${ }^{3}$ When expanded to a given order in $\alpha_{\mathrm{em}}$, the exponential factor

$$
\left(\frac{m_{\gamma}}{\mu}\right)^{\frac{\alpha_{\mathrm{em}}}{2} A_{\beta \alpha}}=\sum_{N=0}^{\infty} \frac{1}{N !}\left(\frac{\alpha_{\mathrm{em}}}{2} A_{\beta \alpha} \ln \frac{m_{\gamma}}{\mu}\right)^{N} .
$$
}

generates logarithmic divergences. At fixed order in the perturbative expansion the transition amplitude does not vanish in the $m_{\gamma} \rightarrow 0$ limit as in the resummed case, on the contrary it diverges logarithmically. This phenomenon may look counterintuitive, but the mathematical mechanism behind it is quite trivial and shows that the perturbative expansion is broken by large calculable IR logarithms. 
the $\Delta E \rightarrow 0$ limit. However at fixed order in the perturbative expansion

$$
\Gamma_{\alpha \beta}\left(\Delta E, E_{T}\right) \stackrel{\Delta E \sim E_{T} \rightarrow 0}{\simeq} \mathscr{N}_{\alpha} F\left(\frac{\Delta E}{E_{T}}, \operatorname{Re} A_{\beta \alpha}\right)\left(1+\alpha_{\mathrm{em}} \operatorname{Re} A_{\beta \alpha} \ln \frac{\Delta E}{\mu}\right)\left|\hat{M}_{\beta \alpha}(\mu)\right|^{2}+O\left(\alpha_{\mathrm{em}}^{2}\right)
$$

the transition rate is logarithmically divergent in the $\Delta E \rightarrow 0$ limit. This divergence is not real, but it signals the breakdown of the perturbative expansion in processes with soft photons in the final state. If we consider for instance the leptonic decay of the hadron $h^{-} \rightarrow \ell^{-}+\bar{v}_{\ell}$, then one can calculate explicitly

$$
A_{\beta \alpha}=\frac{2}{\pi}\left\{\frac{m_{h}^{2}+m_{\ell}^{2}}{m_{h}^{2}-m_{\ell}^{2}} \ln \frac{m_{h}}{m_{\ell}}-1\right\}=\left\{\begin{array}{ll}
2.9(1) & \text { for } \pi^{-} \rightarrow e^{-}+\bar{v}_{e} \\
1.6(1) \times 10^{-2} & \text { for } \pi^{-} \rightarrow \mu^{-}+\bar{v}_{\mu} \\
5.2(1) & \text { for } B^{-} \rightarrow e^{-}+\bar{v}_{e} \\
1.9(1) & \text { for } B^{-} \rightarrow \mu^{-}+\bar{v}_{\mu}
\end{array} .\right.
$$

For a back-of-the-envelope calculation, one can take $\mu$ equal to the largest scale in the problem, i.e. the hadron mass, and calculate $\overline{\Delta E}$ with the property that for all $\Delta E<\overline{\Delta E}$ the $O\left(\alpha_{\mathrm{em}}\right)$ corrections are larger than $10 \%$, i.e.

$$
\overline{\Delta E} \simeq\left\{\begin{array}{ll}
0.5 \mathrm{MeV} & \text { for } \pi^{-} \rightarrow e^{-}+\bar{v}_{e} \\
10^{-362} \mathrm{MeV} & \text { for } \pi^{-} \rightarrow \mu^{-}+\bar{v}_{\mu} \\
400 \mathrm{MeV} & \text { for } B^{-} \rightarrow e^{-}+\bar{v}_{e} \\
3 \mathrm{MeV} & \text { for } B^{-} \rightarrow \mu^{-}+\bar{v}_{\mu}
\end{array} .\right.
$$

Notice that this energy varies a lot with the process. It is obvious that higher-order radiative corrections may become relevant only if large scale separations exists.

\subsection{RM-SOTON calculation}

The RM-SOTON collaboration has presented a strategy and preliminary results for the calculation of the probability rate for the process

$$
h^{-} \rightarrow \ell^{-}+\bar{v}_{\ell}(+\gamma)
$$

with $h=\pi, K$ at order $\alpha_{\mathrm{em}}[15,16,17,18]$. Notice that at this order, at most one photon can be produced in the final state. In infinite volume, the relevant transition amplitudes have the following behaviour in the $m_{\gamma} \rightarrow 0$ limit, which is obtained by expanding eq. (3.4),

$$
\begin{aligned}
& M_{0 \gamma}\left(m_{\gamma}\right)=\left(1+\frac{\alpha_{\mathrm{em}}}{2} A \ln \frac{m_{\gamma}}{\mu}\right) \hat{M}_{0 \gamma}^{(0)}+\alpha_{\mathrm{em}} \hat{M}_{0 \gamma}^{(1)}(\mu)+O\left(\alpha_{\mathrm{em}} m_{\gamma}\right)+O\left(\alpha_{\mathrm{em}}^{2}\right), \\
& M_{1 \gamma}\left(\mathbf{k} ; m_{\gamma}\right)=\alpha_{\mathrm{em}}^{1 / 2} \hat{M}_{1 \gamma}^{(0)}(\mathbf{k})+O\left(\alpha_{\mathrm{em}}^{1 / 2} m_{\gamma}\right)+O\left(\alpha_{\mathrm{em}}^{3 / 2}\right) .
\end{aligned}
$$

As a special case of eq. (3.3), the decay rate in the center-of-mass frame and integrated over the soft photon is

$$
\begin{aligned}
& \Gamma\left(\Delta E, m_{\gamma}\right)=\Gamma_{0 \gamma}\left(m_{\gamma}\right)+\Gamma_{1 \gamma}\left(\Delta E, m_{\gamma}\right)+O\left(\alpha_{\mathrm{em}}^{2}\right), \\
& \Gamma_{0 \gamma}\left(m_{\gamma}\right)=\frac{1}{2 m_{h}}\left|M_{0 \gamma}\left(m_{\gamma}\right)\right|^{2}, \\
& \Gamma_{1 \gamma}\left(\Delta E, m_{\gamma}\right)=\frac{1}{2 m_{h}} \int_{|\mathbf{k}|<\Delta E} \frac{\mathrm{d}^{3} k}{(2 \pi)^{3} 2\left(m_{\gamma}^{2}+\mathbf{k}^{2}\right)^{1 / 2}}\left|M_{1 \gamma}\left(\mathbf{k} ; m_{\gamma}\right)\right|^{2} .
\end{aligned}
$$

The basic idea of the RM-SOTON collaboration is to exploit universality to reshuffle the IR diver- 
gences in a convenient way. Let us consider the auxiliary quantum field theory for hadron, leptons, neutrinos and photons, in which all particles are described as fundamental fields and charged particles are minimally-coupled to photons. In this auxiliary theory the electroweak transition is generated at tree-level by an effective operator of dimension 5 (which can be found in [18]). This auxiliary quantum field theory is usually referred to as point-like approximation, as it could be seen as the leading order of a general effective field theory. The normalization of the effective EW Hamiltonian in the point-like approximation is chosen in such a way that the tree-level amplitude matches the one in the fundamental theory, i.e.

$$
\hat{M}_{\mathrm{PT}, 0 \gamma}^{(0)}=\hat{M}_{0 \gamma}^{(0)},
$$

where the subscript PT denotes quantities calculated within the point-like approximation. Then one defines the structure-dependent (SD) decay rates by subtracting the point-like approximation to the full decay rate, i.e. $\Gamma_{\mathrm{SD}, \star}=\Gamma_{\star}-\Gamma_{\mathrm{PT}, \star}$. In particular

$$
\Gamma\left(\Delta E, m_{\gamma}\right)=\Gamma_{\mathrm{PT}}\left(\Delta E, m_{\gamma}\right)+\Gamma_{\mathrm{SD}, 0 \gamma}\left(m_{\gamma}\right)+\Gamma_{\mathrm{SD}, 1 \gamma}\left(\Delta E, m_{\gamma}\right)+O\left(\alpha_{\mathrm{em}}^{2}\right) .
$$

The crucial observation is that the three terms in the r.h.s. of the above equation are IR finite, and can be calculated separately.

1. The first term in the r.h.s. of eq. (3.20) is IR finite thanks to the Bloch-Nordsieck cancellation mechanism applied to the point-like approximation,

$$
\Gamma_{\mathrm{PT}}(\Delta E) \stackrel{\text { def }}{=} \lim _{m_{\gamma} \rightarrow 0} \Gamma_{\mathrm{PT}}\left(\Delta E, m_{\gamma}\right)<\infty .
$$

Moreover this term is analytically calculable, given the tree-level amplitude, i.e. $F_{h}$.

2. Let us look at second term in the r.h.s. of eq. (3.20). First we notice that a formula, similar to eq. (3.14), holds for the transition amplitude calculated in the point-like approximation. Moreover the radiative function $A$ is the same in the full theory and in the point-like approximation, as it depends only on masses, charges and kinematics. Therefore

$$
\begin{aligned}
\Gamma_{\mathrm{SD}, 0 \gamma} \stackrel{\text { def }}{=} & \lim _{m_{\gamma} \rightarrow 0} \Gamma_{\mathrm{SD}, 0 \gamma}\left(m_{\gamma}\right)=\frac{1}{2 m_{h}} \lim _{m_{\gamma} \rightarrow 0}\left\{\left|M_{0 \gamma}\left(m_{\gamma}\right)\right|^{2}-\left|M_{\mathrm{PT}, 0 \gamma}\left(m_{\gamma}\right)\right|^{2}\right\}= \\
= & \frac{1}{2 m_{h}} \lim _{m_{\gamma} \rightarrow 0}\left(1+\alpha_{\mathrm{em}} \operatorname{Re} A \ln \frac{m_{\gamma}}{\mu}\right)\left\{\left|\hat{M}_{0 \gamma}^{(0)}\right|^{2}-\left|\hat{M}_{\mathrm{PT}, 0 \gamma}^{(0)}\right|^{2}\right\}+ \\
& \quad+\frac{\alpha_{\mathrm{em}}}{m_{h}} \operatorname{Re}\left\{\left[\hat{M}_{0 \gamma}^{(0)}\right]^{*} \hat{M}_{0 \gamma}^{(1)}(\mu)-\left[\hat{M}_{\mathrm{PT}, 0 \gamma}^{(0)}\right]^{*} \hat{M}_{\mathrm{PT}, 0 \gamma}^{(1)}(\mu)\right\}+O\left(\alpha_{\mathrm{em}}^{2}\right)= \\
= & \frac{\alpha_{\mathrm{em}}}{m_{h}} \operatorname{Re}\left[\hat{M}_{0 \gamma}^{(0)}\right]^{*}\left[\hat{M}_{0 \gamma}^{(1)}(\mu)-\hat{M}_{\mathrm{PT}, 0 \gamma}^{(1)}(\mu)\right]+O\left(\alpha_{\mathrm{em}}^{2}\right)<\infty,
\end{aligned}
$$

which shows that $\Gamma_{\mathrm{SD}, 0 \gamma}$ is IR finite as a consequence of the matching condition (3.19). The RM-SOTON collaboration proposes to calculate $\Gamma_{\mathrm{SD}, 0 \gamma}$ from lattice simulations, where the box size $L$ acts as IR regulator. If we denote by $\tilde{\Gamma}_{\mathrm{SD}, 0 \gamma}(L)$ the decay rate in finite volume, the infinite-volume limit is found to be reached up to $1 / L^{2}$ corrections [16]

$$
\tilde{\Gamma}_{\mathrm{SD}, 0 \gamma}(L)=\Gamma_{\mathrm{SD}, 0 \gamma}+O\left(L^{-2}\right) .
$$

This result is nontrivial, as both the point-like and full decay rate have a $1 / L$ term (along with the $\ln L$ IR divergence) whose coefficient is fixed by the gauge Ward identities and general analyticity properties of the effective vertices. ${ }^{4}$

\footnotetext{
${ }^{4}$ In fact this is a nontrivial consequence of the Low and Gell-Mann theorem [46, 47].
} 
3. Finally the third term in the r.h.s. of eq. (3.20) is IR finite since the 1.h.s. of eq. (3.20) is IR finite, i.e.

$$
\Gamma_{\mathrm{SD}, 1 \gamma}(\Delta E) \stackrel{\text { def }}{=} \lim _{m_{\gamma} \rightarrow 0} \Gamma_{\mathrm{SD}, 1 \gamma}\left(\Delta E, m_{\gamma}\right)<\infty .
$$

If $\Delta E$ is asymptotically small, the $\mathrm{PT}$ contribution $\Gamma_{\mathrm{PT}}(\Delta E)$ blows up logarithmically signalling break-down of the perturbative expansion, while the SD contribution $\Gamma_{\mathrm{SD}, 1 \gamma}(\Delta E)$ vanishes linearly. In the case of the pion decay, a window in $\Delta E$ exists such that both the $O\left(\alpha_{\mathrm{em}}^{2} \ln ^{2} \Delta E\right)$ corrections and $\Gamma_{\mathrm{SD}, 1 \gamma}(\Delta E)$ are negligible. It is shown in [15] that reasonable values of $\Delta E$ for which this happens (independently of the lepton in the final state) are in the region of 10-50 $\mathrm{MeV}$. The existence of such window is very much process dependent, and is expected not to hold in processes involving large scale separation because of large logarithms in the coefficients of the $\Delta E$ expansion. In general $\Gamma_{\mathrm{SD}, 1 \gamma}(\Delta E)$ should be calculated from lattice simulations as well.

The preliminary results presented by the RM-SOTON collaboration [17] are obtained in the electroquenched setup (i.e. photons couple only to the valence quarks, but not to the sea quarks), with the $\mathrm{QED}_{\mathrm{L}}$ prescription. The infinite-volume extrapolation of the SD contribution to the decay rate with no photons in the final state has been performed by fitting the coefficient first unknown $L^{-2}$ correction, according to the theoretical analysis presented in [16]. Observables have been calculated with the RM123 method. For the $\pi \rightarrow \mu+v$ process they report for instance

$$
\frac{\Gamma_{\mathrm{NLO}}(\Delta E \simeq 30 \mathrm{MeV})}{\Gamma_{\mathrm{LO}}}=1.0210(15)(\ldots)_{\mathrm{qQED}} .
$$

\subsection{Large collinear logarithms}

The decomposition of decay rates in point-like and structure-dependent contributions is completely general. At fixed order in the perturbative expansion the point-like contribution contains the singular part in $\Delta E$, which is the physical remnant of the soft divergences in the amplitudes. Soft divergences are the only IR divergences in QED if all charged particles are massive. In the limit in which some charged particles become massless new divergences arise. As we will see, these divergences are generated by non-integrable angular singularities in Feynman diagrams, and are therefore referred to as collinear divergences.

The simplest example of collinear divergences is found in the factor $A_{\beta \alpha}$ in eq. (3.11) for the process $h \rightarrow \ell+v$ in the limit $m_{\ell} \rightarrow 0$, i.e.

$$
A_{\beta \alpha} \stackrel{m_{\ell} \ll m_{h}}{\simeq} \frac{2}{\pi} \ln \frac{m_{h}}{m_{\ell}} \rightarrow \infty .
$$

In the real world charged particles are massive. When the considered process involves large scale separations large logarithms arise as a remnant of collinear divergences. The divergence in eq. (3.26) arises from virtual soft photons that are emitted in a direction parallel to the momentum of the hard lepton. However collinear divergences arise also when the photon is not soft, as we will try to argue with a specific example.

Let us consider again the leptonic decay of the hadron $h^{-} \rightarrow \ell^{-}+\bar{v}_{\ell}$. Let $\Gamma_{\mu}(p, k)$ be the effective vertex $h h \gamma$ where $k$ is the photon incoming momentum, $p$ and $-p-k$ are the hadron incoming momenta, and let

$$
\Delta(p)=\frac{Z(p)}{p^{2}+m_{h}^{2}}
$$




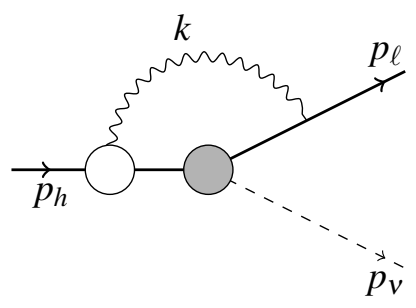

Figure 2: Contribution with one virtual photon to the skeleton expansion of the $h^{-} \rightarrow \ell^{-}+\bar{v}_{\ell}$. The gray circle represents the insertion of the EW effective Hamiltonian, while the white circle represents the effective vertex $h h \gamma$.

be the dressed propagator of the hadron. Let us look at the particular contribution to the transition probability represented in fig. 3.3. This contribution contains the integral

$$
N_{\mu} \int \frac{\mathrm{d} k_{0}}{2 \pi} \int_{\Lambda_{\mathrm{IR}}<|\mathbf{k}|<\Lambda_{\mathrm{UV}}} \frac{\mathrm{d}^{3} k}{(2 \pi)^{3}} \frac{\Gamma_{\mu}\left(\bar{p}_{h}, k\right) Z\left(\bar{p}_{h}+k\right)}{k^{2}\left(2 \bar{p}_{h} \cdot k+k^{2}\right)\left(2 \bar{p}_{\ell} \cdot k+k^{2}\right)},
$$

where Euclidean notation has been used, the on-shell four-momenta are $\bar{p}_{h}=\left(i m_{h}, \mathbf{0}\right)$ and $\bar{p}_{\ell}=$ $\left(i E_{\ell}, \mathbf{p}_{\ell}\right)$, and $N_{\mu}$ is an overall normalization that depends on the kinematic variables, polarizations and the Fermi constant. The IR cutoff has been introduced in order to isolate the hard-photon contribution, and the UV cutoff has been introduced in order to avoid confusion with UV divergences. The integral over $k_{0}$ can be performed by closing the integration path at infinity in the complex plane. One gets contributions from several poles. We are interested in the contribution from either of the photon poles $\bar{k}=( \pm i|\mathbf{k}|, \mathbf{k})$ which is

$$
\frac{N_{\mu}}{8 m_{h}} \int_{\Lambda_{\mathrm{IR}}<|\mathbf{k}|<\Lambda_{\mathrm{UV}}} \frac{\mathrm{d}^{3} k}{(2 \pi)^{3}} \frac{\Gamma_{\mu}\left(\bar{p}_{h}, \bar{k}\right) Z\left(\bar{p}_{h}+\bar{k}\right)}{|\mathbf{k}|^{2}\left(|\mathbf{k}| \sqrt{m_{\ell}^{2}+\mathbf{p}_{\ell}^{2}} \mp \mathbf{p}_{\ell} \mathbf{k}\right)} .
$$

In spherical coordinates with $z=\cos \theta=\mathbf{p}_{\ell} \mathbf{k} /\left(\left|\mathbf{p}_{\ell}\right||\mathbf{k}|\right)$ it becomes apparent that the hadron propagator has a non-integrable singularity at $z= \pm 1$ (the sign depends on which photon pole we have chosen) for $m_{\ell}=0$, which translate into a logarithmic divergence for $m_{\ell}$ small with respect to $\left|\mathbf{p}_{\ell}\right|=\left(m_{h}^{2}-\right.$ $\left.m_{\ell}^{2}\right) /\left(2 m_{h}\right)$, or equivalently $m_{\ell}$ small with respect to $m_{h}$,

$$
\begin{gathered}
\frac{N_{\mu}}{8(2 \pi)^{3} m_{h}\left|\mathbf{p}_{\ell}\right|} \int_{\Lambda_{\mathrm{IR}}}^{\Lambda_{\mathrm{UV}}} \frac{\mathrm{d} k}{k} \int_{0}^{2 \pi} \mathrm{d} \phi \int_{-1}^{1} \mathrm{~d} z \frac{\Gamma_{\mu}\left(\bar{p}_{h}, \bar{k}\right) Z\left(\bar{p}_{h}+\bar{k}\right)}{\sqrt{1+\frac{m_{\ell}^{2}}{\mathbf{p}_{\ell}^{2}}} \mp z} \stackrel{m_{\ell} \ll m_{h}}{\simeq} \\
\simeq \frac{N_{\mu}}{8 \pi^{2} m_{h}^{2}} \int_{\Lambda_{\mathrm{IR}}}^{\Lambda_{\mathrm{UV}}} \frac{\mathrm{d} k}{k}\left[\Gamma_{\mu}\left(\bar{p}_{h}, \bar{k}\right) Z\left(\bar{p}_{h}+\bar{k}\right)\right]_{\hat{\mathbf{k}}= \pm \hat{\mathbf{p}}_{\ell}} \ln \frac{m_{h}}{m_{\ell}} .
\end{gathered}
$$

This formula shows explicitly that the collinear divergence $\ln \left(m_{h} / m_{\ell}\right)$ gets contributions from virtualphotons poles with spatial momentum parallel to the light-lepton momentum but with arbitrary modulus. In particular these contributions are not universal, in the sense that they read the internal structure of the hadron (encoded in the effective vertex and dressed propagator away from the mass-shell).

The presence of quasi collinear divergences is only one of the mechanisms that could enhance the structure-dependent part of the radiative corrections to decay rates of heavy mesons. The existence of resonances slightly heavier than the stable hadron, which can go almost on-shell in radiative processes $[48,26]$ is another possible mechanism. The inherently non-perturbative nature of these phenomena makes their estimate quite difficult. 


\section{References}

[1] A. Duncan, E. Eichten and H. Thacker, Phys. Rev. Lett. 76 (1996) 3894-3897, [hep-lat/9602005].

[2] MILC collaboration, S. Basak et al., PoS CD12 (2013) 030, [1301. 7137].

[3] T. Blum et al., Phys. Rev. D82 (2010) 094508, [1006.1311].

[4] A. Portelli et al., PoS LATTICE2011 (2011) 136, [1201.2787].

[5] S. Borsanyi et al., Science 347 (2015) 1452-1455, [1 406 . 4088 ].

[6] R. Horsley et al., JHEP 04 (2016) 093, [1509.00799].

[7] R. Horsley et al., J. Phys. G43 (2016) 10LT02, [1508. 06401 ].

[8] R. Young, PoS LATTICE2016, 2016.

[9] C. Liu, PoS LATTICE2016, 2016. 1612. 00103.

[10] Z. Fodor et al., Phys. Rev. Lett. 117 (2016) 082001, [1604.07112].

[11] L. Varnhorst, PoS LATTICE2016, 2016.

[12] S. Aoki et al., 1607.00299.

[13] J. L. Rosner, S. Stone and R. S. Van de Water, Sub. to: Particle Data Book (2015) , [1509. 02220].

[14] V. Cirigliano et al., Rev. Mod. Phys. 84 (2012) 399, [1107.6001].

[15] N. Carrasco et al., Phys. Rev. D91 (2015) 074506, [1502.00257].

[16] V. Lubicz et al., 1611.08497.

[17] V. Lubicz et al., PoS LATTICE2016, 2016. 1610.09668.

[18] N. Tantalo et al. 1612.00199.

[19] P. Boyle et al. PoS LATTICE2016, 2016. 1612.05962.

[20] Particle Data Group collaboration, C. Patrignani et al., Chin. Phys. C40 (2016) 100001.

[21] Fermilab Lattice, MilC collaboration, J. A. Bailey et al., Phys. Rev. D89 (2014) 114504, [1403.0635].

[22] BABAR collaboration, B. Aubert et al., Phys. Rev. Lett. 100 (2008) 231803, [0 712 . 3493 ].

[23] CLEO collaboration, N. E. Adam et al., Phys. Rev. D67 (2003) 032001, [hep-ex/ 0210040 ].

[24] BaBar collaboration, B. Aubert et al., Phys. Rev. D79 (2009) 012002, [0 809 . 0828 ].

[25] Heavy Flavor Averaging Group collaboration, Y. Amhis et al., 1207.1158.

[26] F. Bernlochner, PhD thesis, Humboldt U., Berlin, 2011.

[27] N. Tantalo, PoS LATTICE2013 (2014) 007, [1311. 2797].

[28] A. Portelli, PoS LATTICE2014 (2015) 013, [1505. 07057 ].

[29] T. Ishikawa et al., Phys. Rev. Lett. 109 (2012) 072002, [1202.6018].

[30] S. Aoki et al., Phys. Rev. D86 (2012) 034507, [1205.2961].

[31] G. M. de Divitiis et al., JHEP 04 (2012) 124, [1110.6294].

[32] RM123 collaboration, G. M. de Divitiis et al., Phys. Rev. D87 (2013) 114505, [1303. 4896].

[33] M. Gockeler et al., Nucl. Phys. B334 (1990) 527-558.

[34] M. Hayakawa and S. Uno, Prog. Theor. Phys. 120 (2008) 413-441, [0 804.2044 ].

[35] M. G. Endres et al., Phys. Rev. Lett. 117 (2016) 072002, [1507.08916].

[36] L. Polley and U. Wiese, Nucl.Phys. B356 (1991) 629-654.

[37] B. Lucini et al., JHEP 02 (2016) 076, [1509.01636].

[38] Z. Fodor et al., Phys. Lett. B755 (2016) 245-248, [1502 . 06921$].$

[39] M. Creutz, Annals Phys. 117 (1979) 471.

[40] F. Bloch and A. Nordsieck, Phys. Rev. 52 (1937) 54-59.

[41] S. Weinberg, The Quantum Theory of Field, vol. 1. Cambridge University Press, 2005.

[42] D. R. Yennie, S. C. Frautschi and H. Suura, Annals Phys. 13 (1961) 379-452.

[43] G. Grammer, Jr. and D. R. Yennie, Phys. Rev. D8 (1973) 4332-4344.

[44] S. Weinberg, Phys. Rev. 140 (1965) B516-B524.

[45] T. D. Lee and M. Nauenberg, Phys. Rev. 133 (1964) B1549-B1562.

[46] F. Low, Phys.Rev. 96 (1954) 1428-1432.

[47] M. Gell-Mann and M. Goldberger, Phys.Rev. 96 (1954) 1433-1438.

[48] D. Becirevic, B. Haas and E. Kou, Phys. Lett. B681 (2009) 257-263, [0 907 . 1845 ]. 